Les technologies de l'information et de la communication (TIC) dans un processus d'apprentissage organisationnel : pour une coordination émergente entre le local et le global

\title{
Claudine Batazzi-Alexis
}

\section{OpenEdition}

Journals

Édition électronique

URL : http://journals.openedition.org/communicationorganisation/2778

DOI : 10.4000/communicationorganisation. 2778

ISSN : $1775-3546$

Éditeur

Presses universitaires de Bordeaux

Édition imprimée

Date de publication : 1 novembre 2002

ISSN : 1168-5549

\section{Référence électronique}

Claudine Batazzi-Alexis, « Les technologies de l'information et de la communication (TIC) dans un processus d'apprentissage organisationnel : pour une coordination émergente entre le local et le global », Communication et organisation [En ligne], 22 | 2002, mis en ligne le 27 mars 2012, consulté le 21 décembre 2020. URL : http://journals.openedition.org/communicationorganisation/2778 ; DOI : https://doi.org/10.4000/communicationorganisation.2778

Ce document a été généré automatiquement le 21 décembre 2020.

(c) Presses universitaires de Bordeaux 


\title{
Les technologies de l'information et de la communication (TIC) dans un processus d'apprentissage organisationnel : pour une coordination émergente entre le local et le global
}

\author{
Claudine Batazzi-Alexis
}

1 L'introduction massive des TIC (Technologies de l'Information et de la Communication) dans les organisations nous suggère une réflexion d'ensemble sur les processus individuels et collectifs d'acquisition des connaissances. Si la décentralisation des organisations s'est fortement développée au cours des dix dernières années, la littérature managériale laisse présager un renforcement de cette tendance via les réseaux électroniques qui en favorisant la circulation des informations renforceraient les possibilités d'autonomie dans les décisions (Kalika, 2000). D'une manière générale, les frontières des organisations s'estompent tandis que les équipes figées cèdent place à des groupes mouvants et temporaires.

2 L'accroissement de la rapidité de circulation des informations apparait souvent pour les entreprises comme une promesse d'augmentation de leurs profits. Aussi semble-t-on assister parfois à quelque course effrénée à la technologie entre les dirigeants. Le simple fait de posséder des outils technologiques de communication semble considéré par de nombreuses entreprises comme un gage de réussite. On ne peut toutefois qu'être dubitatif face à la vision idyllique (Breton; Proulx, 1996) de certains dirigeants qui prônent la médiation technologique comme moyen d'enrichissement des communications interpersonnelles au sein de l'entreprise. L'idée d'un «monde meilleur» - image vigoureusement dénoncée par Philippe Breton (Breton, 2000) - produite par les TIC est fortement suggérée dans les discours ou écrits sur le sujet. 
3 Les motivations intrinsèques des dirigeants à utiliser les TIC apparaissent pourtant relever davantage d'une volonté d'augmentation de la productivité ou de réduction de coûts aisément identifiables, que de modifications d'ordre structurel difficilement quantifiables (Benghozi; Flichy: d'Iribarne, 2000). L'introduction des TIC dans les échanges de connaissances au sein des organisations suppose pourtant une reconsidération des schèmes organisationnels. De même l'enjeu d'un processus d'apprentissage organisationnel via les TIC ne réside de toute évidence pas dans la technicité des outils mais davantage dans l'usage qui en est fait.

4 Aussi entre une approche techniciste de l'apprentissage (la technique détermine le fonctionnement) et une approche organisationnelle (l'organisation conditionne l'usage), n'est-il pas préférable d'emprunter la voie de l'émergence (Mucchielli, 2000) ? Celle-ci, fruit de l'interaction entre la technologie et l'usage qui en est fait - usage dépendant bien évidemment de nombreux facteurs à l'instar de la personnalité des individus, de leur expérience, de la spécificité de l'entité, de la culture de référence, du style de management...- occasionne indubitablement de nouveaux savoirs.

5 La production de nouveaux savoirs résulterait à la fois de l'interaction homme/machine et des interactions interpersonnelles multipliées par l'abolition des distances. Quant aux échanges des connaissances (Agostinelli, 1999). ils seraient renforcés par la panoplie des TIC mise à la disposition des acteurs comme par exemple les EDI (Echanges de Données Informatisées). Enfin, les TIC participeraient à l'accélération et à la pérennisation du processus de construction de l'identité organisationnelle.

\section{Entre déterminisme technologique et déterminisme organisationnel : la perspective de l'émergence des connaissances}

6 L'enjeu ne paraît plus être aujourd'hui d'estimer l'impact de l'introduction des TIC dans les entreprises mais résiderait davantage dans l'interrogation de l'usage de ces outils par les salariés. N'en déplaise aux postmodernes, Internet ne change pas tant les individus qu'il leur permet de mieux exécuter leurs tâches habituelles (Dahlgren. 2000). Le déterminisme technologique est souvent lié à une vision optimiste des outils où Internet est présenté comme l'élément susceptible de révolutionner l'ordre établi aussi bien au sein de la société que des organisations.

7 Si un déterminisme technologique suppose que les nouvelles technologies sont en mesure d'apporter des changements substantiels dans les organisations, a contrario dans une optique de déterminisme organisationnel, les utilisations des TIC demeurent tributaires de la structure au sein de laquelle elles évoluent. Entre la voie d'un impératif technologique et celle d'un impératif organisationnel, seule la perspective de l'émergence semble correspondre au caractère interdépendant de l'outil et de l'usage (Vaast, 2000).

\section{Utilisation des TIC dans une optique de complémentarité}

8 L'appellation de Technologies de l'Information et de la Communication ne définirait pas exclusivement des outils de communication, aussi sophistiqués soient-ils, mais engloberait la notion de processus social (Levy. 1997) où la pertinence de l'usage prévaudrait sur la technicité de l'outil. La qualité du processus d'appropriation de la 
technique par le salarié demeurant prédominant sur la spécificité de l'outil, la technique et sa mise en œuvre peuvent difficilement être envisagées de façon distincte.

Aussi, l'optique pour les organisations de développer via les TIC un processus d'apprentissage organisationnel ne réside-t-elle pas de toute évidence dans l'évaluation des qualités ou des défaillances des outils mis à leur disposition mais davantage dans leurs modes de fonctionnement. Ainsi des études de type monographique menées sur une vingtaine d'entreprises (Benghozi; Flichy.; d'Iribarne, op.cit.) ont conclu à une diversification des applications d'Internet (assimilé à un modèle hybride) en fonction de la spécificité des entreprises.

10 Ainsi, le courrier électronique trouve aisément sa place auprès du courrier papier et $d u$ téléphone même si on constate encore certaines réticences de la part des entreprises dans l'envoi par voie électronique des documents officiels (Benghozi; Flichy: d'Iribarne, op. cit.). Dans ce cas là, le courrier papier double généralement le courrier électronique. De même, l'enjeu d'un groupware (Le Bœuf. 2002) n'est pas de se substituer aux échanges physiques ou téléphoniques mais de devenir un outil complémentaire d'échanges de connaissances entre les salariés.

11 La formation en ligne (ou e-learning) s'envisagerait aussi dans une optique de complémentarité à la "formation en présence " soit en amont d'une formation dispensée par un formateur, soit en aval (à l'issue de la période de formation). Cette nécessaire complémentarité se retrouve également dans le télétravail où le " présentiel » s'adjoint $\mathrm{au}$ « distanciel » (Guillaume, 2000).

12 Ainsi le fait d'imaginer que l'introduction des TIC au sein des organisations est susceptible de remplacer de façon radicale les relations interpersonnelles revient à s'allier aux technophobes et craindre une paupérisation des contacts humains directs. La mise en commun des différents outils et de leurs usages favoriserait alors la création de connaissances nouvelles même si le cadre contextuel diffère quelque peu notamment par la reconsidération des notions de temps et d'espace

\section{Vers un déplacement de l'interaction}

13 Si comme nous l'avons évoqué les outils communicationnels sont davantage appréhendés dans une optique de renforcement plutôt que de substitution, les interactions entre les différents éléments du système n'en sont que plus nombreuses et variées. Les TIC remettent toutefois en cause la représentation du réel en redéfinissant les notions de temps et d'espace

La perspective de l'émergence suppose en effet que les caractéristiques respectives des outils et des situations d'entreprise soient prises simultanément en considération dans une approche dynamique (Vaast, op.cit.). Le réseau Intranet influerait sur le fonctionnement d'une organisation tout en demeurant le construit de cette dernière. Il s'agit bien là d'un processus émergent, co-construit par la technicité de l'outil, l'usage qui est fait et dans une vision plus large par le contexte.

La particularité de l'interaction via les TIC réside toutefois dans le caractère asynchrone de l'interaction et dans la médiation par l'outil électronique. Les interactions se développent au sein d'un cadre de participation de co-présence médiatisé et asynchrone (Beaudoin : Velkovska, 1999) qui se déplace en créant des regroupements d'individus dans un cadre professionnel mais en dehors des locaux de l'entreprise. S'il est relativement 
aisé de reconnaître grâce à la médiation de certains outils, des phénomènes d'interaction entre des individus éloignés géographiquement. d'autres outils développant l'asynchronisme amènent toutefois quelques interrogations sur la nature même des relations interpersonnelles et de l'interaction (Batazzi ; El Idrissi, 2002).

En effet, il est aisé de reconnaitre le phénomène d'interaction via des réseaux électroniques de caractère synchrone comme les Internet Relay Chat (IRC) ou par le biais de la visio-conférence et même du téléphone, mais il est plus difficile à admettre sur des réseaux asynchrones comme les messageries électroniques, les forums de discussion... Les réseaux asynchrones s'inscrivent pourtant au sein d'un cadre de participation semblable à celui défini par Goffman (1974). dans la mesure où le partage des représentations est susceptible de s'effectuer. Il s'agit bien de la "création d'un espace commun d'intercompréhension" (Beaudoin; Velkovska, op. cit.). Bien évidemment, la définition d'un tel espace impose aux acteurs certaines contraintes liées initialement à l'interprétation puis aux comportements qui en résultent. Les relations sociales médiatisées sont déterminées à la fois par les relations existantes entre les acteurs et par les transformations engendrées par les interactions. Et c'est seulement à partir des représentations partagées par les individus que l'organisation est en mesure d'élaborer des savoirs collectifs. On assiste bien là à la production de nouvelles connaissances. Si la première phase d'un processus d'apprentissage organisationnel (la création de connaissances nouvelles) peut aisément être explicitée à la fois à travers la complémentarité des outils et les phénomènes d'interaction, qu'en est-il de la deuxième phase du processus à savoir la transformation des connaissances individuelles en compétences organisationnelles?

\section{De la notion de connaissance à celle de compétence organisationnelle : les TIC dans le processus de transfert des savoirs.}

17 Les réflexions sur le thème de l'apprentissage organisationnel ne sont pas récentes. En effet, dans les années vingt, Max WEBER avait déjà mis en exergue l'enjeu d'un apprentissage collectif en avançant que les bureaucraties étaient capables d'apprendre d'après leurs propres expériences. Des chercheurs en organisation comme Cyert, March, Weick, Simon et Cohen se sont également penchés sur le concept d'apprentissage organisationnel. Ainsi Cyert et March ont posé en 1963 l'apprentissage des « routines » en tant que processus organisationnel fondamental et Weick de son côté en 1979 a tenté d'établir une comparaison systématique entre la psychologie classique de l'apprentissage individuel et celle de l'apprentissage organisationnel (Cohen; Sproull. 1991). Quant à Simon, il a essayé de transposer les concepts de la psychologie cognitive à l'apprentissage organisationnel (1979).

La gestion des savoirs diffère du simple traitement d'informations car elle suppose une reconsidération de l'organisation du travail. Les entreprises souhaitant obtenir des compétences organisationnelles sont ainsi obligées de se pencher sur la formalisation des connaissances individuelles. Comment peuvent-elles en effet insérer dans les routines organisationnelles, les règles implicites et les savoir latents? Le transfert des connaissances ne repose pas exclusivement sur des connaissances formelles et explicites mais comprend aussi des connaissances tacites, par définition difficiles à cerner et à 
déterminer ${ }^{1}$. Aussi la difficulté réside-t-elle essentiellement dans le transfert de ces connaissances d'un état informel vers un état formel afin d'être en mesure de les faire partager par les autres membres tout en les inscrivant dans les processus de l'organisation.

La notion de compétence en englobant celle de connaissance constitue de ce fait le stade le plus perfectionné de la chaîne de connaissances. Il se produit un processus de coconstruction entre la compétence et l'action (Mira-Bonnardel S.. 2000) Ainsi les TIC par la création de cadres d'échanges et d'apprentissages participeraient à la socialisation des connaissances tacites et de ce fait faciliteraient la transformation d'une connaissance individuelle en compétence organisationnelle.

De l'acquisition individuelle des connaissances...

21 Un apprentissage est qualifié de comportemental lorsque les individus par des processus réitérés d'essai-erreur apprennent des gestes et des comportements en vue de parfaire ou de développer des savoir-faire. Dans un contexte d'apprentissage de type cognitif, l'apprentissage du sujet repose sur la construction de nouveaux schémas de pensée.

22 L'utilisation des TIC par les organisations s'inscrit dans un double processus d'apprentissage : celui de l'usage de la technologie encore appelé «navigation » et celui de la distanciation critique qui peut être définie comme la sélection des informations utiles pour l'utilisateur parmi la masse informationnelle. Nous faisons ici notamment référence à la hiérarchie des apprentissages proposée par Bateson (Bateson. 1981). Ainsi si le premier niveau correspond à un apprentissage de type mécaniste et automatique, le second niveau relève quant à lui davantage de l'ordre cognitif. Enfin le troisième niveau apparaît comme l'ultime niveau de l'apprentissage dans la mesure où l'individu est capable de créer lui-même le cadre de l'apprentissage.

Les interdépendances dépassent toutefois rapidement le caractère fonctionnel et les participants impliqués dans le réseau le font vivre et le développent. Il s'agit d'un apprentissage « en marchant » qui se réaliserait « de façon quelque peu désordonnée et au fil des découvertes "(Alexis, 1999). La difficulté réside alors dans l'articulation des savoirs individuels au sein d'un mécanisme collectif de mémorisation. Aussi les TIC et plus particulièrement les réseaux numériques apparaissent-ils propices dans la formalisation puis dans l'échange de compétences.

24 ...à un processus collectif d'articulation des compétences

25 Les réseaux électroniques en facilitant le traitement et la mémorisation des savoirs individuels constitueraient de toute évidence un outil de production et de gestion des connaissances dont l'ensemble dépasserait la somme des capacités individuelles des acteurs. Ainsi Argyrys et Schon (1978) ont proposé une hiérarchie des apprentissages semblable à celle de Bateson mais adaptée aux organisations où sont définies les acceptions «d'apprentissage en simple boucle » et « d'apprentissage en double boucle ». Ainsi «l'apprentissage en simple boucle» est limité à la correction des écarts par l'organisation elle-même entre la situation présente et la situation souhaitée : le schéma de référence ne subissant aucune modification. Il s'agit davantage d'un phénomène d'adaptation que de l'émergence d'un réel processus d'apprentissage. L'apprentissage en « double boucle » se forme quant à lui à travers la reconsidération des modèles mentaux et structurels, les normes organisationnelles étant alors révisées.

26 Une simple modification du comportement des individus au sein d'une organisation relève d'un apprentissage en simple boucle. On évoque l'apprentissage en double boucle 
lorsque les individus sont amenés à s'interroger sur la signification de leurs comportements. Une organisation susceptible de produire de façon permanente des «apprentissages en double boucle» renforcerait son potentiel d'actions stratégiques (Normann, 1985). L'interrogation demeure toutefois quant à l'apport des TIC dans le passage d'un état d'apprentissage dit « en simple boucle » à un état qualifié de " double boucle ».

27 À l'instar de tout événement, les TIC lors de leur introduction dans l'organisation amorcent inévitablement un processus d'apprentissage organisationnel, les salariés étant placés en situation de changement et d'instabilité. À l'issue de cette phase d'adaptation s'instaure un processus de stabilisation des savoirs où les représentations sont mises en commun et les pratiques, partagées. La dynamique du système réside essentiellement, à ce stade du processus, dans la pertinence des savoirs échangés ainsi que dans la fiabilité des transferts interpersonnels.

28 Accessibles par des systèmes Intranet, les groupwares développent par exemple au sein des entreprises de nouveaux modes de coordination et de collaboration entre les individus notamment dans la gestion des savoirs en adjoignant aux échanges, la mémorisation. En effet les réseaux facilitent la structuration des connaissances de chaque salarié en créant des routines collectives ${ }^{2}$. Le travail coopératif assisté par ordinateur étant pratiqué à travers les conférences électroniques, le téléchargement de fichiers... le développement d'un processus d'apprentissage organisationnel ne peut être pensé que dans une stratégie d'avantage concurrentiel durable (création de valeur par les réseaux électroniques).

Aucune organisation ne peut toutefois se résumer au recensement des savoirs formels. Même dans les entreprises tayloriennes fortement formalisées, les routines de travail comprenaient des savoirs tacites développés en parallèle par les salariés. La difficulté du transfert des connaissances individuelles en savoirs collectifs réside par conséquent dans la formalisation de connaissances qui ne sont souvent que des pratiques individuelles. La simple verbalisation de ces savoirs par leurs détenteurs relève parfois de l'exploit (Dostaler : Boiral, 2000).

30 La compétence collective définie comme la résultante d'un processus interactif entre d'une part des connaissances formalisées et d'autre part des savoirs tacites, devient un système évolutif de connaissances partagées et appliquées par les membres de l'organisation. On peut suggérer le concept « d'organisation apprenante », c'est-à-dire celui d'une organisation susceptible d'encourager la formation de tels apprentissages et d'assurer la pérennité des compétences organisationnelles (Guilhon; Trepo, 2000). L'articulation des connaissances consistant en leur codification et leur transfert, les TIC apparaîtraient alors comme un puissant levier dans le transfert des représentations individuelles vers un niveau collectif simultanément par les échanges de connaissances et la formalisation nécessaire de ces échanges.

31 Une formalisation exagérément poussée des connaissances risque toutefois d'engendrer un effet de paupérisation quant elle évince une part importante du contexte. Aussi tout traitement d'information doit-il être assorti d'un processus narratif qui positionne l'information dans son contexte (Belmondo. 2000). La transformation d'une connaissance individuelle en compétence organisationnelle suppose donc l'entretien d'une culture de partage. 


\section{L'inscription des compétences dans les schèmes organisationnels via les TIC}

32 Un phénomène d'interaction ne peut être compris qu'à travers la prise en compte d'un système culturel plus vaste. L'interaction entre deux individus ne se résume pas en effet à un jeu de questions et de réponses. Des schémas communs d'interprétation se créent par les savoirs et les relations qu'entretiennent les individus entre eux. Ces schémas peuvent être assimilés à de complexes mélanges d'actions et de processus de mémorisation qui interviennent simultanément au niveau individuel et au niveau collectif. Reflets des caractéristiques de l'environnement, de la société, de l'organisation.... ils sont négociés en permanence entre les acteurs (Belmondo, op.cit.). Ces processus de négociation ou d'ajustement s'apparentent au phénomène de construction d'une identité organisationnelle.

33 Si la pérennité des organisations repose sur leur capacité à actualiser et à développer les savoirs collectifs, nous suggérons que les TIC contribuent indubitablement à l'inscription de ces savoirs dans l'identité organisationnelle. Les TIC s'avéreraient toutefois pertinentes dans le fonctionnement d'une entité seulement lorsque le terrain serait propice «(..) la véritable modernité est d'abord politique et, seulement ensuite, éventuellement technique " (Breton, 1997). Ainsi les TIC en participant à la création du lien social autoriseraient à envisager le partage des compétences davantage dans l'optique d'une réalité à construire que dans l'utopie d'un idéal collectiviste.

\section{Les TIC dans la construction de l'identité organisationnelle}

Dans un contexte de changement organisationnel à l'instar de celui produit par l'introduction des réseaux numériques (Internet. Intranet...), les dirigeants transitent d'une vision cybernétique de l'organisation (régulation en environnement stable) à une vision biologique (l'organisation produit des connaissances dans un contexte en fluctuation). Ainsi une approche organique de l'entité déplace la notion d'identité située (connaissance initiale que possèdent les acteurs les uns des autres) vers celle d'identité discursive (construite au cours du jeu des questions et réponses c'est-à-dire dans l'interaction) (Beaudoin; Velkovska, 1999). On glisse d'un changement prescrit (rationalité substantive) à un changement construit où l'individu bâtit l'organisation (rationalité procédurale ou plutôt processuelle).

Selon la théorie de l'action située de Schuman (1987). les actions menées par les membres d'une organisation ne suivent pas une logique de déroulement mais s'inscrivent au contraire au sein d'interactions locales parmi les éléments contextuels. Les travaux d'Orlikowski (2000) précisent davantage le phénomène de co-construction du système en reconnaissant que la technologie, l'action humaine et la situation se génèrent de façon interdépendante et conjointement. En effet, contrairement aux recherches liées à la théorie de la structuration qui isolent la technologie pour la positionner en relation avec le système organisationnel (Groleau, 2001). Orlikowski souligne l'impossibilité de dissocier la technologie de son usage et de son contexte. On assiste donc à un processus de co-construction entre les TIC et l'identité organisationnelle. Aussi la découverte de l'identité d'une organisation a-t-elle intérêt à s'effectuer selon la démarche minutieuse 
d'un anthropologue, par la prise en compte des éléments contextuels susceptibles d'orienter l'usage des TIC.

Si l'identité organisationnelle peut être définie comme un ensemble complexe de représentations mentales, les auteurs américains distinguent toutefois la notion d'identité organisationnelle de celle de culture d'entreprise. Ainsi l'identité correspondrait bien à un schéma mental résultant d'une construction de sens tandis que la culture d'entreprise demeurerait le fruit de l'action des dirigeants lié notamment à des phénomènes de mode. D'autres auteurs ont repris la même idée en distinguant les notions "d'identité professée " et "d'identité ressentie» (Soenen: Moingeon, 2000). Si l'identité professée est celle désirée par les dirigeants, l'identité ressentie correspond quant à elle à une représentation collective de l'organisation partagée par ses membres.

D'une manière générale, l'identité d'une organisation est formée à la fois par des composantes formelles telles que sa structure ou ses modes de fonctionnement et par des éléments informels comme par exemple le vécu collectif. Ainsi les règles tacites de comportement qualifiées de "routines organisationnelles " peuvent s'avérer bénéfiques ou au contraire néfastes au processus d'apprentissage et par extension à tout changement organisationnel. Le vécu d'une organisation n'est pas sans influence sur son avenir. Il détermine entre autres sa capacité d'adaptation et de renouvellement des savoirs. La théorie de la structuration de Giddens [1987] avance que des propriétés structurelles se dégagent à travers les actions quotidiennes des individus et ces dernières guident bien évidemment les actions futures (Groleau. op. cit.).

Une question s'impose toutefois à ce stade de notre réflexion à savoir celle liée à la découverte de la motivation des membres d'une organisation à accepter de se dessaisir d'une partie de leurs savoirs personnels au profit d'une collectivité qu'ils ont souvent du mal à se représenter. Comment les TIC peuvent-elles contribuer à l'élaboration d'un lien entre le local et le global ? Par ailleurs, existe-t-il réellement des actions concertées entre les acteurs? En d'autres termes, est-ce que les individus agissent au sein des entreprises de façon régulée? Face à ces interrogations, les communautés de pratique seraient susceptibles de fournir des éléments de réponse.

\section{Renforcement des communautés de pratique par les TIC}

Les savoirs que l'on peut qualifier d'interconnectés supposent toutefois d'être inscrits sur des supports pour devenir accessibles à tous et ne pas demeurer la propriété exclusive de ceux qui les ont créés. Les TIC prennent là une dimension nouvelle : celle de jouer les relais dans le processus d'apprentissage en inscrivant dans l'identité de l'organisation de nouveaux comportements. Bien que l'instrumentation apparaisse ici comme nécessaire, elle nécessite toutefois d'être envisagée au sein d'une réflexion globale prenant en considération le caractère indivisible de l'outil et de son usage.

Les notions de local et de global demeurent toutefois théoriques. La complexité de leurs observations réside notamment dans le rôle de celui qui l'apprécie. Il est toutefois possible de considérer le local et le global comme les deux dimensions d'une même réalité. On qualifie ainsi de "projection réciproque» (Autissier. 2000) la relation entre le local et le global dans la mesure où le local communique au global les éléments qu'il perçoit et le global les positionne dans leur contexte. On aboutit à une émergence de la coordination où la projection réciproque du local et du global contextualise et formalise les actions individuelles pour maintenir la coordination des actions collectives (Autissier. 
op. cit.). Dans une perspective interactionniste le système au sein duquel se positionnent le local et le global créé de nouveaux patterns et de nouvelles relations.

41 Le local et global requièrent des modes de coordination susceptibles de construire des consensus. Ainsi les acteurs construisent une représentation collective de leurs actions qui leur permet de se positionner les uns par rapport aux autres. Aussi la coordination ne peut-elle qu'émerger des combinaisons entre le local et le global c'est-à-dire entre les stratégies individuelles et les stratégies collectives qui visent au contraire à minimiser les comportements opportunistes.

42 Notre réflexion porte par conséquent sur l'enjeu des TIC comme éléments de coordination susceptibles de favoriser les interactions entre le local et le global et par extension la médiation des savoirs au sein des organisations. La coordination regroupe des tâches qui ne sont pas directement liées à la production mais sans lesquelles la production ne pourrait être exécutée dans des conditions satisfaisantes. Les dispositifs de coordination via les réseaux numériques permettraient notamment aux individus d'adopter des comportements similaires à ceux qu'ils pourraient avoir s'ils étaient physiquement en présence.

43 Par conséquent si nous admettons que chaque individu dans un élargissement de l'autonomie poursuit son propre objectif, les dispositifs de coordination permettent alors de développer les capacités individuelles des acteurs et de créer une synergie (Autissier. op. cit.). On assiste ainsi dans une perspective de construction du changement, à un réel processus de structuration qui émerge des initiatives individuelles notamment à travers "des communautés de pratique» (Grimand. 2000). Ces dernières ne peuvent être assimilées à des groupes de projets, la différence résidant essentiellement dans le non engagement des membres autour d'un objectif commun. La vocation de ces communautés de pratique consiste davantage en la création de valeur par et pour leurs membres.

Le caractère informel des communautés de pratique en favorisant les interactions permet aux individus de bâtir un cadre d'exploration des savoirs. Ces communautés apparaissent particulièrement fécondes dans le transfert des apprentissages individuels vers des apprentissages collectifs. Si la majorité des travaux sur l'apprentissage organisationnel dissocie le savoir de ses usages, les communautés de pratique au contraire les associent dans la tripartite savoir-usage-émergence, en participant notamment au renouvellement des routines organisationnelles. Entre comportements spontanés et comportements délibérés de transfert et de partage des connaissances, l'organisation devient simultanément le contexte et le produit de ses actions (Guilhon; Trepo, op.cit.). Les communautés de pratique seraient à cet effet en mesure d'inclure les connaissances individuelles dans les connaissances organisationnelles.

En renforçant la réactivité des entreprises à la fois par une collaboration élargie à un nombre plus important d'individus et par une réactualisation accélérée des connaissances, les formations de type e-learning apparaissent comme un complément pertinent dans la pérennité $\mathrm{d}$ un processus d'apprentissage organisationnel (CaronFasan: Janissek-Muniz. 2002.). Les TIC en s'insérant dans le fonctionnement d'une organisation, constitueraient alors un élément interdépendant des autres éléments du système (les individus, la structure, l'historique...) dans le processus d'apprentissage organisationnel.

46 Il serait toutefois préjudiciable de confondre les communautés de pratique avec de simples réseaux de relations interpersonnelles. Les pratiques ne peuvent en effet se 
constituer que dans le partage des histoires individuelles et collectives. Les TIC participent à la coordination des individus au sein des communautés de pratique en renforçant leurs possibilités d'échange et de partage des représentations. On assiste bien là à un phénomène de co-construction des savoirs entre le local (l'individu ou un groupe d'individus) et le global (l'organisation dans son ensemble).

\section{Des éléments de conclusion}

$\mathrm{Au}$ cours de cette réflexion, nous avons essayé d'apprécier les changements induits par l'introduction des TIC dans les processus d'apprentissage des organisations. Ainsi l'apprentissage organisationnel ne peut être circonscrit à des actions délibérées telles que la formation, la veille stratégique ou même le e-learning mais s'envisage davantage dans une approche systémique de l'organisation où les savoirs collectifs émergent à travers les pratiques et les expériences accumulées.

Loin d'être exhaustive la présentation des usages a tenté d'apporter un éclairage complémentaire sur les nouvelles formes de travail suscitées par un développement d'outils tels que Internet, Intranet, système de visioconférence, groupware... Des travaux plus approfondis relatifs à l'appréciation de la volonté des acteurs à coopérer ainsi que leur capacité à s'approprier la technologie s'avéreraient pertinents. De même certaines questions relatives à l'attitude des dirigeants dans la mise en œuvre d'un tel processus nécessiteraient d'être étudiées. C'est en effet de toute évidence le dirigeant ou dans une vision plus large le manager, qui décide de donner une dimension organisationnel le aux connaissances développées localement. Celui-ci a l'obligation de développer une représentation collective dans le souci de fédérer les individus autour de valeurs. Identité ou culture, le débat ne se pose plus ici. les deux se mêlant aisément. Où commence la volonté d'un dirigeant d'imposer sa vision et où s'achève sa capacité à gérer la coconstruction d'un système?

\section{BIBLIOGRAPHIE}

AGOSTINELLI, S, La construction d'un espace collectif de communication, in Comment penser la communication des connaissances, sous la direction de Serge Agostinelli, Ed. L'Harmattan. 1999.

ALEXIS, $\mathrm{H}$; Les Hypermedias dans l'entreprise : une opportunité d'apprentissage, in Comment penser la communication des connaissances - $d u$ CD rom à l'Internet, sous la direction de Serge Agostinelli, Ed. L'Harmattan. Paris: 1999.

ARGYRYS, C., SCHON, D, Organizational learning: A theory of action perspective. Addison Wesley, 1978.

AUTISSIER. D., Des artefacts informationnels pour une coordination émergente. Colloque AIMS, les 24, 25 et 26, Montpellier. 2000.

BATAZZI. C. EL IDRISSI, D., La stratégie e-business : véritable révolution ou simple évolution?. Colloque AIM. Affaire électronique et société de savoir : opportunités et défis, Hammamet, Tunisia, 2002. 
BATESON. G., La nouvelle communication. Ed. du Seuil : Paris, 1981.

BEAUDOIN, V., VELKOVSKA, J, Constitution d'un espace de communication sur Internet, in Réseaux № 97, Hermès Science Publications. 1999.

BELMONDO. C, L'articulation entre outils de gestion et connaissances tacites/explicites et leur intégration dans la gestion des connaissances. Colloque AIMS, les 24.25 et 26. Montpellier, 2000.

BENGHOZI. P.J., FLICHY, P., IRIBARNE. A, Le développement des NTIC dans les entreprises françaises, in Réseaux $N^{\circ} 104$. Hermès. Science Publications, 2000.

BRETON. P., Le culte de l'Internet - Une menace pour le lien social. Ed. La Découverte : Paris. 2000. BRETON, P, PROULX, S, L'explosion de la communication. La Découverte : Paris, 1996.

CARON. M.L., JANISSEK. R., Pérennisation de l'intelligence collective anticipative : le e-learning comme solution. Colloque AIM, Affaire électronique et société de savoir : opportunités et défis. Hammamet, Tunisia, 2002.

COHEN. D.M., SPROULL. L, Editors'Introduction, in Organisation Science. vol. 2 Nº 1. February, 1991. CYERT, R., MARCH. J, A behavioural theory of the firm, Englewood Cliffs, N.J., Prentice Hall, 1963.

DOSTALER, I., BOIRAL. O. Mobiliser les connaissances tacites : l'exemple d'un atelier d'assemblage électronique. Colloque AIMS, les 24, 25 et 26, Montpellier. 2000.

GOFFMAN, E., Les rites d'interaction. Les Editions de Minuit : Paris, 1974.

GRIMAND. A., Le rôle des acteurs et des dispositifs de gestion dans la construction des apprentissages stratégiques, Colloque AIMS, les 24, 25 et 26, Montpellier, 2000.

GROLEAU. C, Les dimensions matérielles et sociales du travail collectif : les défis posés par les technologies d'information-communication. Journée d'Etudes Org \& Co, Castres, 7,8 et 9 juin. (2001). GUILHON, A., TREPO, G., La compétence collective : le chaînon manquant entre la stratégie et la gestion des ressources humaines, Colloque AIMS, les 24, 25 et 26, Montpellier. 2000.

GUILLAUME, M., La maîtrise virtuelle de l'espace réel, in Réseaux $N^{\circ} 100$, Hermès Science Publications. 2000.

KALIKA. M., Le management est mort, vive le e-management. Revue Française de Gestion, $\mathrm{N}^{\circ} 129$, Juin-Juillet-Août. 2000.

LEBOEUF, C, Le groupware dans la complexité, in La fin du groupware? Résurgence d'une dynamique organisationnelle assistée par ordinateur, sous la direction de Claude Leboeuf. Ed. l'Harmattan, 2002.

LEVY, P., Cybercuiture, Ed. Odile Jacob, 1997.

MIRA-BONNARDEL. S., Pour un management conjoint des connaissances et des compétences. Colloque AIMS, les 24.25 et 26, Montpellier, 2000.

MUCCHIELLI, A., La nouvelle communication. Ed. Armand Colin, 2000.

NORMANN. R., Developing Capabilities for organizational learning, in Organizational Strategy and Change by Johannes M. Pennings and Associates. Ed. Jossey: London, 1985.

ORLIKOWSKI. W., Using technology and constituting structures: a practice lens for studying technology in organizations. Organization Science, $11 \mathrm{~N}^{\circ} 4,2000$.

SCHUMAN, L., I'lans and Situated Action: The problem of Human-Machine Communication. Cambridge University Press. 1987.

SIMON, H., Information processus models of cognition?, in Annual Review of Psychologie, N 30. 1979. 
SOENEN, G., MOINGEON, B., Les identités de l'entreprise : intégrer les apports du marketing, de la théorie des organisations et du management stratégique. Colloque AIMS, les 24, 25 et 26.

Montpellier, 2000.

VAAST, E., Intranet et aléas organisationnels. in Réseaux $N^{\circ} 104$. Hermès Science Publications. 2000.

\section{NOTES}

1. Tacite vient du latin « tacitum » et signifie ce qui est caché et secret.

2. Le terme de "routines» a été utilisé en 1995 par Argyrys. En 1988. Levit et March avait employé celui de " procédures standards opérationnelles ».

\section{RÉSUMÉS}

La capacité des organisations devient cruciale dans un environnement où l'obsolescence des connaissances est accélérée par la vitesse de diffusion de l'innovation. Au-delà du renouvellement obligatoire des connaissances individuelles du personnel, les organisations se trouvent dans l'obligation, face notamment à l'avancée exponentielle du progrès technologique, de susciter la mise en place de processus permanents d'apprentissage.

The organisations ability is all the more important in today's environment since the knowledge becomes outdated increasingly fast with the diffusion of innovation. More than just renewing the individual knowledge of the staff, the organisations as they face an exponential advance of technological progress, do have to incite the setting up of permanent learning methods.

\section{INDEX}

Mots-clés : apprentissage organisationnel, communautés de pratique

\section{AUTEUR}

\section{CLAUDINE BATAZZI-ALEXIS}

L'auteur, Docteur en Sciences de Gestion et qualifié en Sciences de l'Information et de la Communication apporte un double éclairage sur les phénomènes de changement organisationnel. Ses travaux de recherche sur l'usage des Technologies de l'Information et de la Communication dans les organisations ainsi que son expérience en entreprise l'ont amené à gérer des processus d'apprentissage organisationnel. 